Check for updates

Cite this: RSC Adv., 2018, 8, 22659

\title{
Molecular modelling of supramolecular one dimensional polymers
}

\author{
Divya B. Korlepara (D) a and S. Balasubramanian (iD)*b \\ Supramolecular polymers exemplify the need to employ several computational techniques to study \\ processes and phenomena occuring at varied length and time scales. Electronic processes, \\ conformational and configurational excitations of small aggregates of chromophoric molecules, solvent \\ effects under realistic thermodynamic conditions and mesoscale morphologies are some of the \\ challenges which demand hierarchical modelling approaches. This review focusses on one-dimensional \\ supramolecular polymers, the mechanism of self-assembly of monomers in polar and non-polar \\ solvents and properties they exhibit. Directions for future work are as well outlined.
}

Received 20th April 2018

Accepted 11th June 2018

DOI: $10.1039 / \mathrm{c} 8 \mathrm{ra} 03402 \mathrm{~h}$

rsc.li/rsc-advances

elongation process is governed by another equilibrium

\section{Introduction}

The term supramolecule was introduced by Karl Lothar Wolf et al. in $1937 .{ }^{1}$ Chemistry beyond the molecule, based on noncovalent interactions is known as supramolecular chemistry. $^{2-4}$ Supramolecular polymers are large-scale (meso, micro) structures arising out of weak non-covalent interactions between molecules in solution. These interactions are directional and are reversible at near-ambient conditions such as hydrogen bonding, $\pi-\pi$, electrostatic, donor-acceptor, hydrophobic attractions and metal-ion coordination. Such polymers form spontaneously from a solution of monomers beyond a critical concentration and below a particular temperature. Commercial exploitation of supramolecular polymers use the advantage of reversibility and responsiveness, specifically in adhesives, printing, cosmetics, personal care, and coatings ${ }^{5-7}$ and in many bio-medical applications. ${ }^{8-20}$

The mechanism of self-assembly of molecules in solution can be broadly classified into two categories based on the dependence of the association constant $K$, between species on oligomer size. ${ }^{21}$ These are isodesmic and cooperative mechanisms. ${ }^{22,23}$ As the name suggests, in the isodesmic mechanism (or equal- $K$ model) the association constant is independent of the size of the oligomer. ${ }^{24}$ The degree of polymerisation is found to be broad in an isodesmic process, even under favourable thermodynamic conditions. On the other hand, the cooperative mechanism of self-assembly also known as the nucleationelongation process, possesses two or more different equilibrium constants with oligomer size. The formation of the nuclei is associated by a nucleation equilibrium constant $\left(K_{\mathrm{n}}\right)$, and the

${ }^{a}$ Chemistry and Physics of Materials Unit, Jawaharlal Nehru Centre for Advanced Scientific Research, Bangalore, India

${ }^{b}$ Chemistry and Physics of Materials Unit, Jawaharlal Nehru Centre for Advanced Scientific Research, Bangalore, India. E-mail: jncbala@gmail.com; Fax: +91 802208 2766; Tel: +91-8022082808 constant, $\left(K_{\mathrm{e}}\right)$. Supramolecular polymers formed via such mechanisms can be topologically 1-dimensional, ${ }^{25-27}$ 2-dimensional $^{28-32}$ or 3-dimensional structures. ${ }^{33-38}$ In the current review, we limit ourselves to modelling studies of onedimensional supramolecular polymers as these constitute the majority of theoretical/computational studies in the literature.

Organic molecules which self-assemble to form 1-d supramolecular polymers, in general, have three components, (see schematic in Fig. 1(a)): an aromatic planar core, a selfassembling moiety and the two connected by a linker which can be used to provide functionality to the molecule. The selfassembling moiety will usually contain an alkyl group or groups bulkier than an alkyl (such as, say, cholesterol). The optimal inter-alkyl distance between neighbouring molecules in a 1-dimensional supramolecular polymer is around $4.2 \AA$, while the corresponding aromatic cores prefer to be located at distances of around 3.5 ̊. In order to meet both these needs, the polymer exhibits a helical twist shown in Fig. 1(b). ${ }^{39-43}$

Chirality is often a central theme in supramolecular polymers, as a preferred helical handedness of the polymer can be used as an experimental probe via circular dichroism spectroscopy. ${ }^{44}$ While achiral molecules will form 1-d stacks of either handedness in equal proportions, stacks formed out of

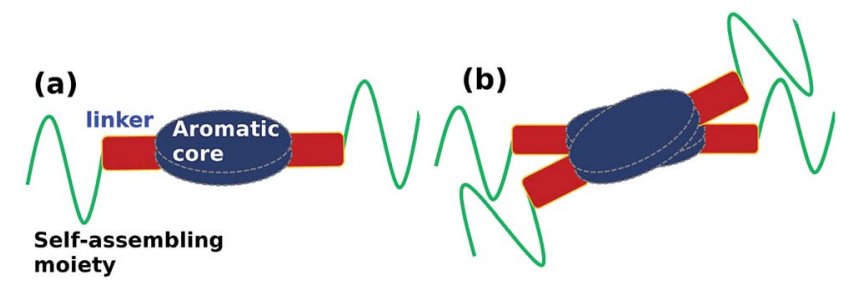

Fig. 1 (a) Schematic representation of key moieties of a monomer $\&$ its (b) dimer, exhibiting a helical twist. 
molecules with a chiral center exhibit a preference of one particular helical sense. ${ }^{44}$ The position of the chiral center in the self-assembling moiety is seen to determine not only the helical sense of the supramolecular stack, ${ }^{\mathbf{4 4 , 4 5}}$ but also influences its stability. The solvent too plays an important role on the conformation of the molecule in a stack. They can intercalate into the stacks, thereby influencing its conformation. ${ }^{\mathbf{4 6 7}}$ The orientation of functional groups, primarily in the linker moiety, can give rise to several low-lying geometries of the monomer, the existence of which can be obtained from crystallographic studies. $^{48-50}$

Superstructures formed by 1-d supramolecular polymers are to a large extent, fibers. These fibers show dynamic selfassembly and dis-assembly in response to change in environment, which are the advantages of supramolecular polymers over the traditional ones. Thus, in this review, we focus on modelling studies of one dimensional polymers, ${ }^{48,51-55}$ a topic which has been reviewed recently by Bochicchio and Pavan recently. ${ }^{55}$ The experimental interest in these materials is from optoelectronic applications point of view. UV-Vis absorption and fluorescence spectroscopy are the primary experimental tools employed. These spectra can in turn be calculated for single molecules as well as for very short oligomers in gas phase using quantum chemical calculations. The process of oligomerization can be studied using all-atom molecular dynamics simulations, while nano and meso scale structure and organization can be investigated using coarse grain methods. Formation of fibers from polymers and their morphologies can be studied using continuum methods. The length and time scales associated with these processes and techniques are illustrated in Fig. 2.

\section{One dimensional (1D) polymers}

\subsection{Gas phase calculations}

2.1.1 Monomers. Benzene-1,3,5-tricarboxamide (BTA) is a well known molecule in supramolecular polymers, due to its

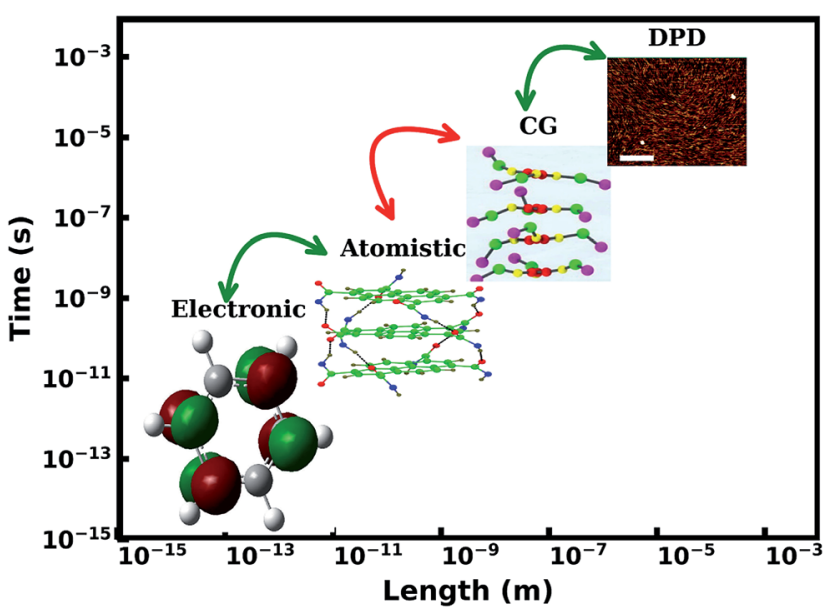

Fig. 2 Hierarchy of multiscale modeling of supramolecular polymers. This figure has been reproduced from ref. 56 and 57 with permission from the American Chemical Society. DPD = dissipative particle dynamics, CG $=$ coarse-grained. ease of synthesis and wide variety of applications. ${ }^{4,59-66}$ While its aromatic core is a simple phenyl ring, the linker is an amide group possessing an electric dipole moment arising out of the large electronegativity difference between the $\mathrm{N}-\mathrm{H}$ hydrogen and the carbonyl oxygen. The structure of the molecule is shown in Fig. 3(a). The core-linker portion of the BTA single molecule has a planar geometry in gas phase. The three amide groups are coplanar and their dipole vectors cancel, thus making the net dipole moment of single molecule BTA to be zero. ${ }^{58}$ While BTA is planar, a few other molecules which have been studied for supramolecular polymerization, such as [2.2]paracyclophane ${ }^{48}$ (Fig. 3(b)) and pyrene derivative ${ }^{50}$ (Fig. 4) are non-planar in the ground state and exhibit interesting dipole configurations as low-lying geometrically excited states (Fig. 5 and 6) and their dipole moment values are tabulated in Table 1.

2.1.2 Oligomers. In the case of BTA, oligomerization proceeds via the formation of intermolecular amide-amide hydrogen bonds which would necessitate the amide group of each molecule to orient away from the benzene core plane. Three intermolecular hydrogen bonds per molecule are formed and an oligomer thus contains three hydrogen bond helices, each with a pitch of six molecules long. As BTA is achiral, the winding of these helices can be either left- or right-handed and an equal proportion of such stacks are observed in solution. A BTA dimer can be formed in either of two ways - one in which all the three hydrogen bond dipole vectors are oriented in the same direction (3:0 state) and another in which any one of the dipole vectors is flipped ( $2: 1$ state). The latter is the ground state, as can be rationalized on electrostatic grounds and the same has been observed in quantum chemical calculations as well as force field calculations (Fig. 7). The $2: 1$ state is also thermodynamically stabilized on entropic grounds, as it can be generated in three distinct ways. The longer the oligomer, the greater is the contribution of this entropic stabilization to the $2: 1$ state. As the net dipole moment of every molecule in a stack is aligned along the same direction, the stack acquires a large electric dipole moment, called the 'macrodipole'. Futhermore, as the solvent, in general, is non-polar, such macrodipoles of stacks present in solution interact with each other without much dielectric screening. The richness of dipole orientational landscape found in BTA oligomers is further enhanced in oligomers of PCpTA and PCA, as those isolated molecule themselves exhibit many dipole configurations (Fig. 5 and 6).

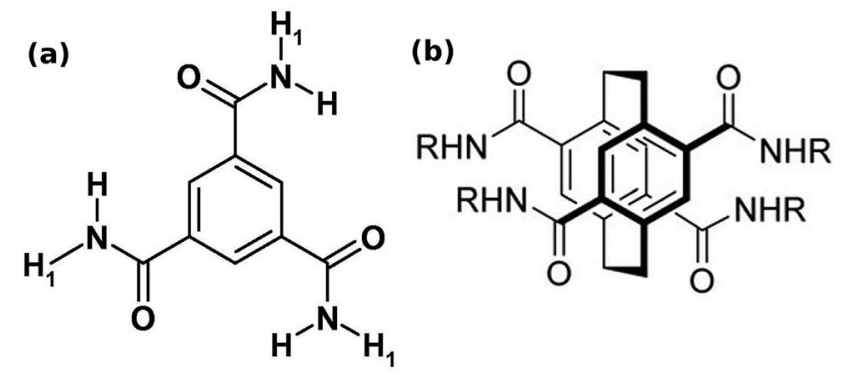

Fig. 3 (a) Benzene-1,3,5-tricarboxamide (BTA). This figure has been reproduced from ref. 58 with permission from Elsevier. (b) $p C p-$ $4,7,12,15$-tetracarboxamide ( $p C p T A), R=H$. This figure has been reproduced from ref. 48 with permission from Wiley- $\mathrm{VCH}$. 
(a)

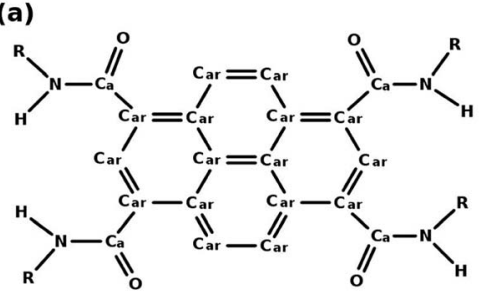

(b)

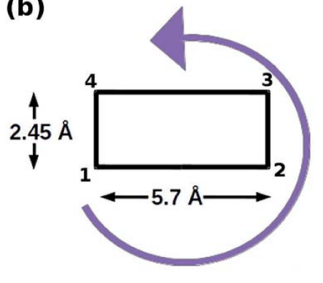

Fig. 4 (a) Pyrene derivative (PCA), $\mathrm{R}=\mathrm{H}$, (b) its schematic representation. This figure has been reproduced from ref. 50 with permission from the American Chemical Society.

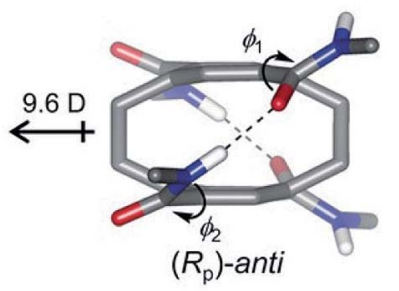

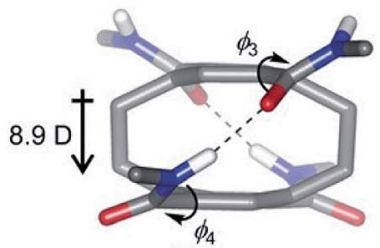

$\left(R_{\mathrm{p}}\right)$-syn
Fig. 5 Two conformations of pCpTA, $\mathrm{R}=\mathrm{CH}_{3}$ and the values of dipole moments are also shown. This figure has been reproduced from ref. 48 with permission from Wiley-VCH.

A wide variety of molecules with different functional groups have been synthesized and studied experimentally for their polymerization mechanism. ${ }^{67,68}$ However, a rationalization of the mechanism exhibited by a given molecule based on its molecular characteristics has eluded researchers for a long time. Based on a careful analysis of molecular functional groups and gas phase quantum chemical calculations of short oligomers, Kulkarni et al. proposed that molecules which possess functional groups that enable the formation of a macrodipole (or in general, one that leads to a long range interaction) along the stacking direction lead to a cooperative mechanism of selfassembly while those which lack such characteristics selfassemble via the isodesmic mechanism. ${ }^{58}$ Gas phase calculations of binding energies (B.E.) of oligomers for BTA reveal their cooperativity (Fig. 8), where
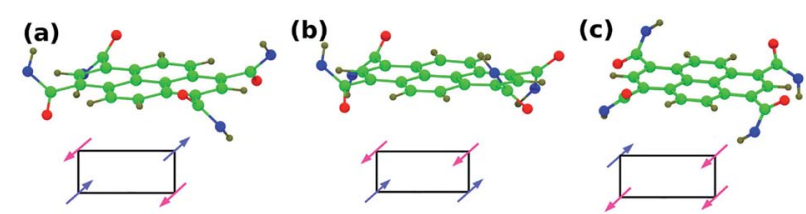

(d)

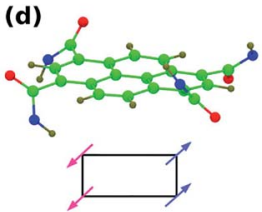

(e)

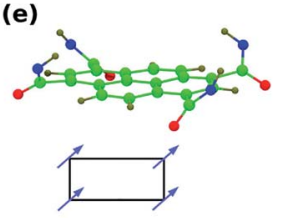

(f)

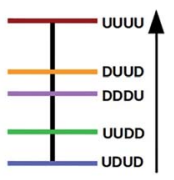

Fig. 6 (a-e) PCA configurations, $\mathrm{R}=\mathrm{CH}_{3}$. Symbols ' $U$ ' \& ' $\mathrm{D}$ ' represent the dipole orientation about the aromatic core to be up and down respectively. Use the arrow guide in Fig. 4b. Panel (f) are the energies of the configurations.

Table 1 Dipole moments of monomers of BTA, pCpTA, PCA

\begin{tabular}{lll}
\hline Molecule & Configuration & Dipole moment $(D)$ \\
\hline BTA & Planar & 0.491 \\
pCpTA & Anti & 9.60 \\
& Syn & 8.90 \\
PCA & Non-planar/UDUD & 0.27 \\
& Non-planar/UUDD & 0.00 \\
& Non-planar/DDUU & 4.75 \\
& Non-planar/DUUD & 0.00 \\
& Non-planar/UUUU & 9.61
\end{tabular}

$$
\text { Binding energy }=\frac{E_{n}-\left(n E_{1}\right)}{n-1}
$$

2.1.3 Optical properties. Supramolecular polymers are well studied for their optoelectronic properties. Although the experiments which determine the same are carried out either in solution or in thin film forms of the samples, the corresponding computations have been carried out only in gas phase as electronic structure calculations in other media are computationally challenging.

If the system under consideration has electron deficient and electron rich molecules, then they can interact via charge transfer interaction. One of the well known electron deficient molecule is naphthalene diimide (NDI) whose structure is shown in Fig. 9(a). NDI can form ground state charge transfer complexes with electron-rich aromatic solvents such as benzene, $o$-xylene and mesitylene, one of which is shown in Fig. $9(b)$.

With an increase in the ability of the solvent molecule to donate electrons, its interaction with NDI increases as exemplified by the vertical transition wavelengths in Fig. 10 calculated through time dependent DFT (TDDFT) methods on clusters of NDI with different solvent molecules. This work demonstrated that care must be taken in the choice of aromatic

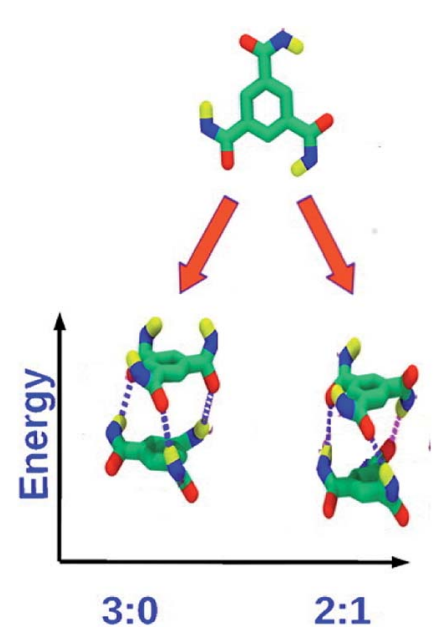

Fig. 7 Top: monomer of BTA with $\mathrm{R}=\mathrm{H}$. Bottom: dimer configurations. The $2: 1$ dipole configuration is the ground state. This figure has been reproduced from ref. 49 with permission from the American Chemical Society. 
(a)

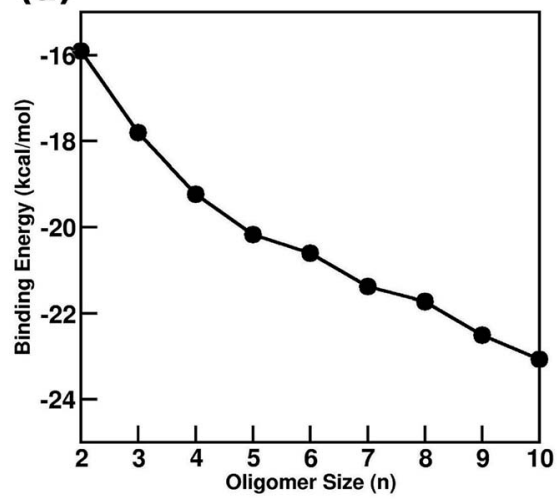

(b)



Fig. 8 (a) Binding energy (BE) of BTA obtained from quantum calculations using PBE method with dispersion corrections (b) optimized configuration of a BTA hexamer with electrostatic potential map illustrating the emergence of a macro dipoles. This figure has been reproduced from ref. 58 with permission from Elsevier.

solvents for NDI derivatives. ${ }^{69}$ The energy level diagram and molecular orbitals involved in the CT transition of NDI coordinated by electron rich mesitylene molecules is shown in Fig. 11.

Density functional theory calculations also demonstrated a $\sigma$ bond formation between Pd metal centres in a oligophenyleneethynylene (OPE) based Pd(II) pyridyl complex which polymerized via both supramolecular and metallophilic interactions. ${ }^{70}$

While gas phase and cluster calculations can be used to identify intermolecular interactions which contribute to molecular association, the mechanism of self-assembly and larger scale structures can be understood only via calculations carried out including explicit solvent molecules, at finite temperature, under bulk conditions. Due to computational challenges, a full-blown DFT based simulation is not possible and has not been reported in the literature; it is not warranted either. Thus, empirical force field based MD simulations are well suited to answer these questions and these have been carried out with either polar and non-polar solvents.

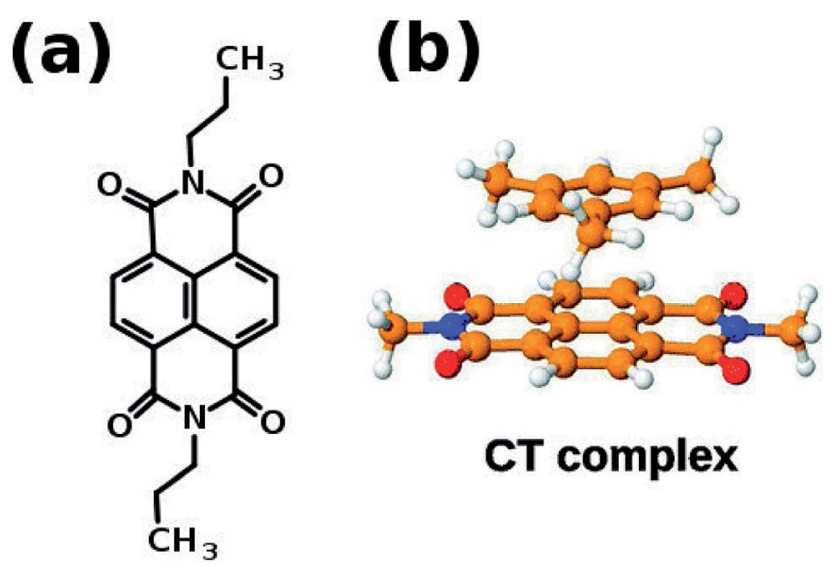

Fig. 9 (a) Naphthalene diimide (NDI) derivative $1^{\prime}$ (b) schematic representation of charge transfer complex. This figure has been reproduced from ref. 69 with permission from the PCCP Owner Societies.



Fig. 10 Calculated vertical transitions of NDI derivative-solvent complexes at the BLYP-D3/DZVP//B3LYP/6-31+G(d,p) level of theory ( $\mathrm{fwhm}=1000 \mathrm{~cm}^{-1}$ ). The black dashed arrow indicates the changes in the CT transition with increasing electron donating capacity of the solvent molecule. This figure has been reproduced from ref. 69 with permission from the PCCP Owner Societies.

\section{Molecular dynamics simulations}

\subsection{All atom (AA) simulations}

3.1.1 Non-polar solvents. Atomistic MD simulations with realistic interatomic potentials can capture a significant part of the nucleation and self-assembly of organic molecules in solution. MD simulations have shown the self-assembly of BTA in liquid $n$-nonane to form long stacks wherein the amide dipoles are arranged in the $2: 1$ configuration. ${ }^{49}$ Using advanced sampling methods, the dimerization free energy of BTA in solution has been calculated to be $-12.81 \mathrm{kcal} \mathrm{mol}^{-1}{ }^{49}$ The free

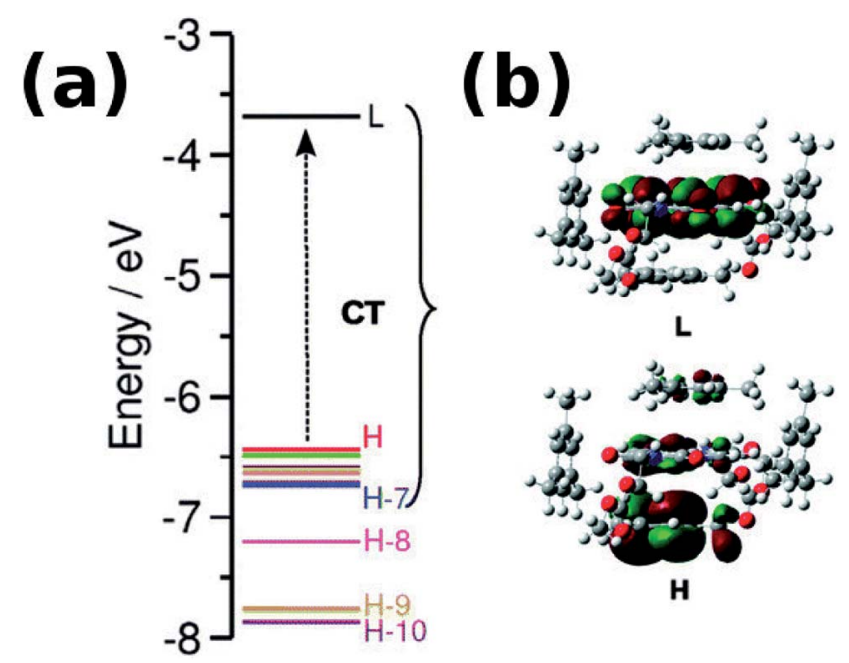

Fig. 11 (a) Computed energy level diagram of $1^{\prime}$ with four molecules of mesitylene. (b) Molecular orbitals of $1^{\prime}+$ four molecules of mesitylene. $\mathrm{H}$ and $\mathrm{L}$ stand for $\mathrm{HOMO}$ and LUMO respectively. Reproduced from ref. 69 with permission from the PCCP Owner Societies. 
energy change involved in the formation of long oligomers from shorter ones has also been calculated using these simulations which demonstrate the cooperative nature of self-assembly of BTA, consistent with experiments. Both BTA and PCA form long fibers in non-polar solvents.

With small modifications in the functional groups, it is possible to have molecules to either polymerize or not. This was exemplified in a system eerily similar to BTA. Three molecules of a ester-based BTA family ${ }^{71}$ are shown in Fig. 12. These molecules have both amide (A) and carboxylate (C) functional groups, thus one can have the possibility of forming either three (AA type) or six (AC type) intermolecular hydrogen bonds between a pair of molecules. Gas phase quantum chemical calculations showed that a AC dimer was more stable than a AA one by $20 \mathrm{kcal} \mathrm{mol}^{-1}$. However the AC dimer cannot elongate to form a polymer as it is left with no 'free' hydrogen bonding sites. MD simulations $\mathrm{s}^{72}$ rationalized the experimental observations of the capability of BTA-Met and BTA-Phe to form long polymers and that of BTA-Nle to form just dimers. The oligomers of the former two molecules are further stabilized by weak $\mathrm{C}-\mathrm{H} \cdots \mathrm{S}$ and $\mathrm{C}-\mathrm{H} \cdots \pi$ interactions respectively.

Supramolecular polymers have a wide range of applications as they can form gels, nano fibers, rod like structures and liquid crystalline (LC) materials. ${ }^{50,52,57,73-75}$ Both BTA and PCA exhibit a LC phase at room-temperature and have been studied for their ferroelectric behaviour experimentally. ${ }^{52,64,65,76,77}$ Atomistic MD simulations revealed that BTA in its LC phase displays a reversal of handedness of the hydrogen bond helices upon reversal of electric field applied in a fashion akin to that in ferroelectric experiments. ${ }^{78}$ However, such a handedness reversal was not observed in the case of PCA; $;^{50}$ an electric field reversal caused the formation of bifurcated intermolecular hydrogen bonds in the latter. The difference in the behavior of these two systems was attributed to the difference in the rotational symmetry of the core of these two molecules. While BTA is $\mathrm{C}_{3}$ symmetric,

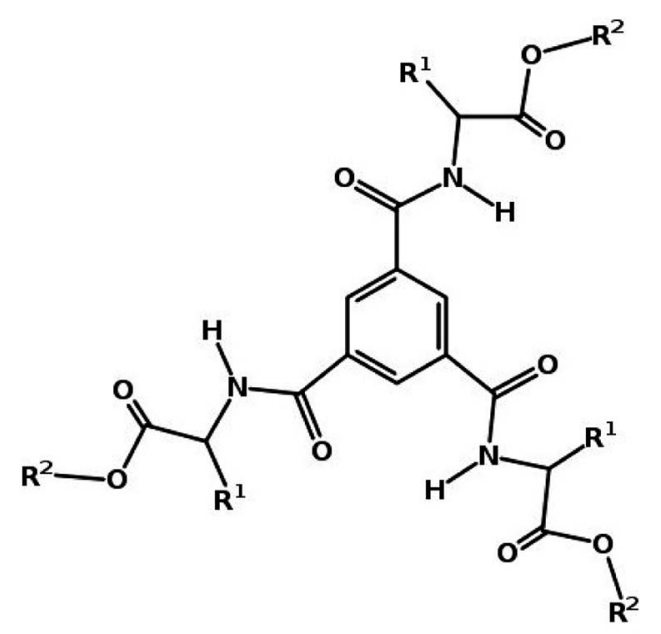

$$
\begin{gathered}
\text { BTA-Met } \mathbf{R}^{1}=(\mathrm{S})-\mathrm{CH}_{2} \mathrm{CH}_{2} \mathrm{SCH}_{3} \\
\text { BTA-Nle } \mathbf{R}^{1}=(\mathrm{S})-\mathrm{CH}_{2} \mathrm{CH}_{2} \mathrm{CH}_{2} \mathrm{CH}_{3} \\
\text { BTA-Phe } \mathbf{R}^{1}=(\mathrm{S})-\mathrm{CH}_{2} \mathrm{C}_{6} \mathrm{H}_{5} \\
\mathbf{R}^{2}=\text { dodecyl }
\end{gathered}
$$

Fig. 12 Ester based BTA structures. ${ }^{71}$
PCA is $\mathrm{C}_{2}$ symmetric which leads to unequal amide-amide near neighbor distances within a molecule. Thus, we generalize that handedness reversal of hydrogen bond helices in a supramolecular stack in its LC phase upon switching of electric field will happen in systems with either $\mathrm{C}_{3}$ or $\mathrm{C}_{4}$ symmetry in their core.

Atomistic MD simulations ${ }^{50}$ have also revealed yet another interesting difference between BTA and PCA in their LC phases: their dipolar relaxation mechanisms. At equilibrium (zero external field), the asymmetric dipole configuration is the ground state. The approach to this ground state from a dipole excited state proceeds in a sequential fashion (i.e., the dipole of one molecule flips, followed by its neighbor and so on) in the case of a stack of PCA, while it is found to be random in the case of BTA. The difference in behaviour can be attributed to the difference in dihedral barriers of the two molecules. These intricate details are not directly accessible to experiments, making MD simulations a valuable complementary tool. MD simulations were also able to rationalize results of a beautiful experiment on solvent intercalation within the self-assembling moiety which results in the ability to thermodynamically switch the helical sense of a supramolecular stack. Solvent molecules intercalate into the stack's side chains at low temperature and leave them at higher temperatures leading to a reversal of helical sense of the stack. ${ }^{47}$

The conjecture that long range interactions (such as dipoledipole) along the stacking direction leads to cooperative selfassembly was convincingly demonstrated ${ }^{79}$ in a family of compounds based on Perylene-3,4,5,10-tetracarboxylic acid bisimides (PBIs) which are shown in Fig. 13. The compounds illustrated therein differ either in their linker or in their selfassembling moiety. While molecules 1 and 4 have a carbonate linker (possessing an electric dipole moment), molecule 3 has a polar ether group, devoid of dipole moment. The selfassembling moiety in 1 and 3 are cholesterol, while in 4 , it is



Fig. $13 \mathrm{PBI}$ derivatives and their mechanisms of self-assembly. This figure has been reproduced from ref. 79 with permission from the American Chemical Society. 
a chiral swallow tail (CST), branched alkyl group. Molecule 1 followed a cooperative mechanism of self-assembly while both 3 and 4 followed an isodesmic pathway. The cholesteric group provides rigidity while the CST in 4 makes the molecule fluxional and occupy a larger volume, thus destroying the helical order required for self-assembly. The presence of a carbonate group provides the needed dipole-dipole interaction for cooperativity which, along with the order-inducing cholesterol group in 1 leads to a large macrodipole moment along the stack direction. These observations from atomistic MD simulations (see Fig. 14) were corroborated from dielectric spectroscopy experiments as well.

Enhanced sampling methods. While atomistic MD simulations are able to exhibit the aggregation of monomers from solution to form short oligomers, they are unable to capture the elongation of such oligomers to longer stacks due to 'entropic bottleneck', i.e., the volume of configuration space spanned by dispersed monomers is so vast (in simulations carried out at experimentally realistic concentrations of the chromophore), that longer oligomers are not formed within the timescales of atomistic MD simulations. Furthermore, even the short oligomers (say, of length 6-10 molecules) exhibit defects (metastable states) which would need a long time to reach the more stable, helically ordered state. If one has to retain atomistic interactions and yet obtain thermodynamically stable structures, one needs to employ enhanced sampling methods. ${ }^{80-86}$ Replica exchange (or parallel tempering) and replica exchange solute tempering (REST) are some of the methods which have been employed in the area of supramolecular polymer modelling. By utilizing these techniques, one can obtain highly ordered polymer structures with helical H-bonding pattern as shown in Fig. 15. The REST (or REST2) (8,86 $^{\mathbf{8}}$ methods temper just the solute molecules and not the solvent and thus are more efficient in

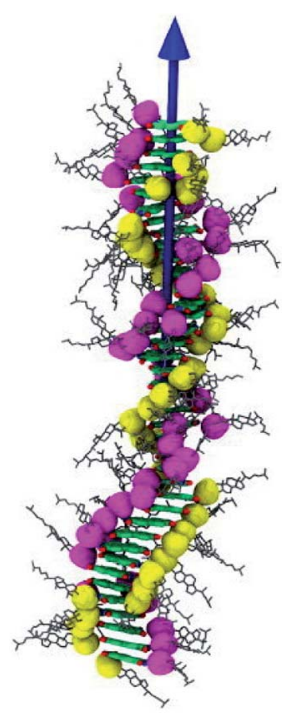

1

4.70

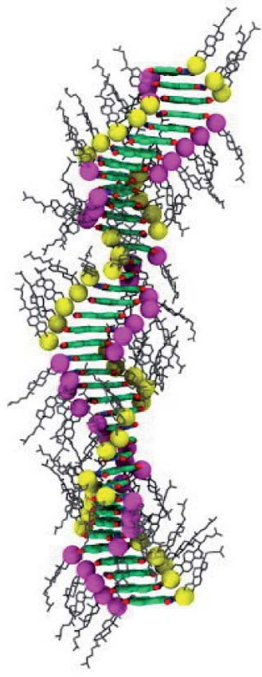

3

$-2.20 \mathrm{D}$

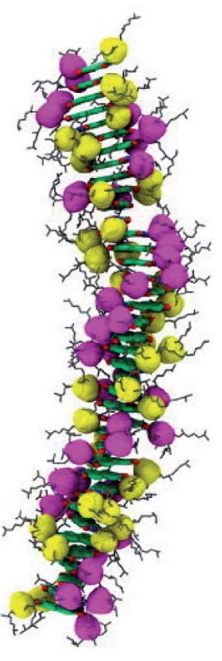

4

$-1.43 \mathrm{D}$
Fig. 14 Snapshots from MD simulations of PBI derivatives and their dipole moments. This figure has been reproduced from ref. 79 with permission from the American Chemical Society.

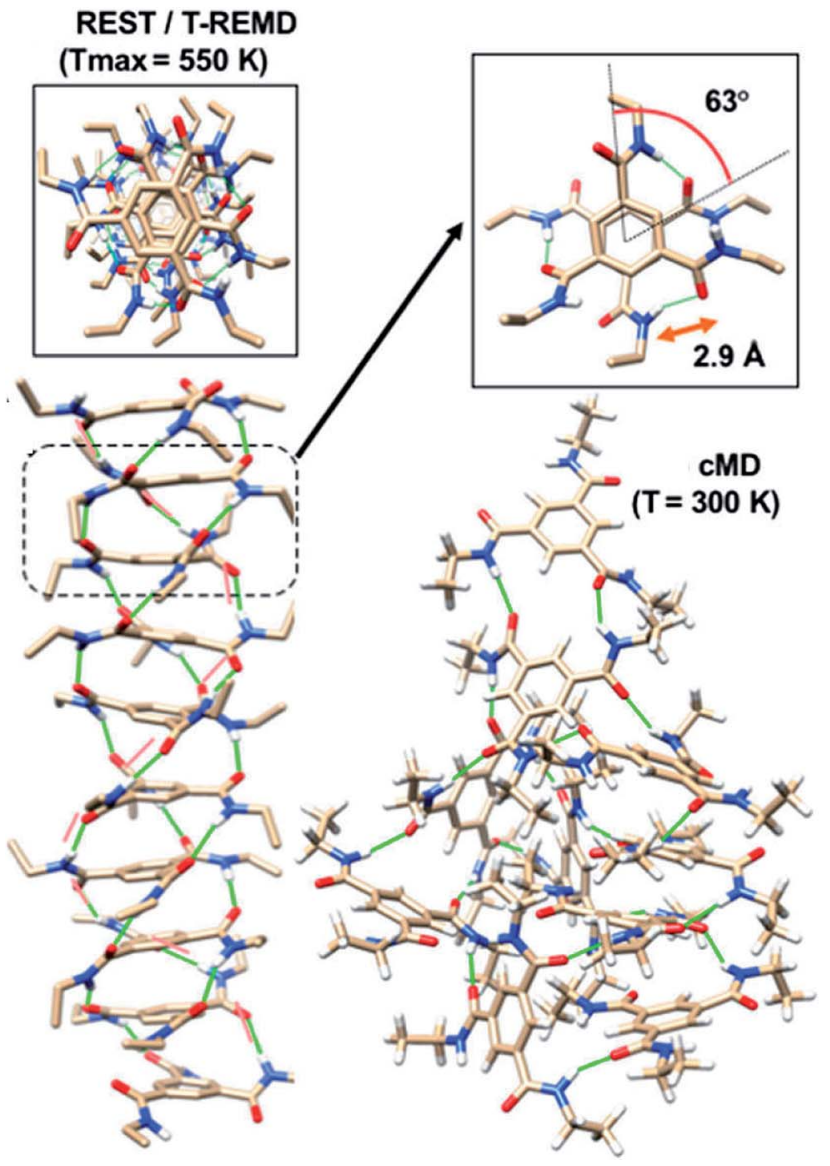

Fig. 15 Self-assembled structures from common MD (cMD), T-REMD and REST at different values of $T_{\text {max }}$. This figure has been reproduced from ref. 87 with permission from the American Institute of Physics.

sampling the configuration space of the chromophore molecules than the simpler replica exchange method (T-REMD) ${ }^{87}$

3.1.2 Polar solvents. Supramolecular polymers have been investigated in aqueous solutions as well, whose importance can be gauged by the fact of their use in regenerative medicines. ${ }^{88}$ The core of these molecules being aromatic, their water solubility is poor; to enhance the same, the self-assembling moiety is made polar typically by adding ethylene glycol groups. Such molecules with a BTA core are called as BTE and a few of them ${ }^{89-91}$ are shown in Fig. 16. While the fibrous morphology of both chiral and achiral water soluble BTA polymers were similar, their UV-Vis absorption spectra showed a difference, arising from the difference in packing of the core in stacks. MD simulations were performed on preformed stacks of both chiral and achiral BTA soaked in water. ${ }^{92}$ While the polymer constructed from the chiral molecule retained its helicity, the achiral one did not; the former was attributed to the larger barrier for dihedral rotation arising out of the chiral centre. In the case of the achiral molecule, the intermolecular hydrogen bond broke easily to interact with water, which led to differences in the degree of packing of BTA cores.

The cooperative nature of aggregation of BTE in water was studied using atomistic MD simulations. ${ }^{92}$ Intermolecular hydrogen bonding was identified as a major contributor to the 


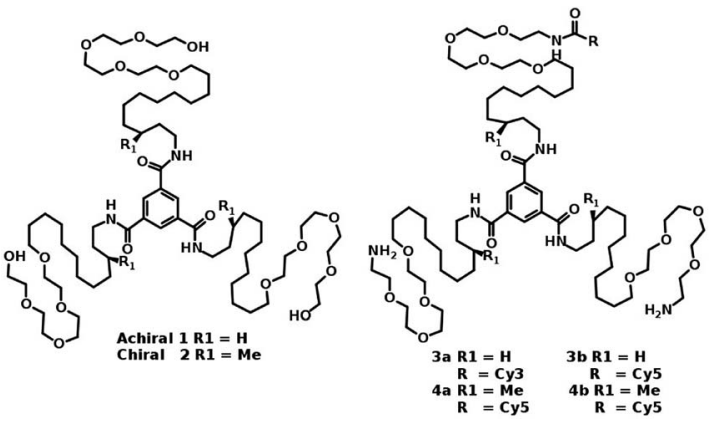

Fig. 16 Molecular structures of water soluble chiral and achiral BTA. ${ }^{91}$

stability of the supramolecular polymer. When the hydrogen bonding ability of the monomer was turned off synthetically (as well as in computation see Fig. 17), the ability to form supramolecular stacks was considerably reduced. Thus, the directional driving force of hydrogen bonding was attributed as the underlying reason for cooperativity. ${ }^{92}$

Biomimetic self-assembly driven by an external fuel is one of the long standing problems in supramolecular polymerization. Recently, a oligo(p-phenylenevinylene) derivative (OPV) was found to be selective to ATP over other triphosphates such as UTP, CTP, or GTP. Atomistic MD simulations showed that the ATP-bound OPV oligomers were stabilized by weak hydrogen bonding interactions which were absent in other triphosphates. During the process of self-assembly, the removal of a slip between the OPV core planes with increasing oligomer size was observed and this process explained the experimentally observed lag time in circular dichroism (CD) and UV/Vis spectra. $^{93}$

The assembly/disassembly of microtubules in biological systems is activated by chemical fuel which triggers conformational transitions in the molecules constituting the tubule. Researchers have worked on synthetic mimics where the conformational transition is triggered photochromically, using the well known system of cis-trans isomerization in azo group based compounds. ${ }^{94,95}$ Self-assembled microtubule-like structures with hydrophobic regions buried inside the tubule, exposing the hydrophilic part of the molecules to the solvent (water) have been synthesized. The azo group is present within

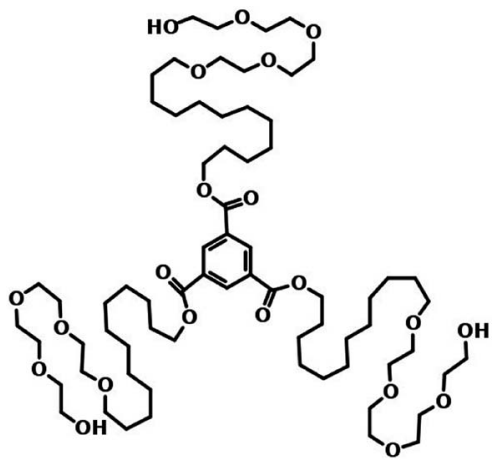

Fig. 17 Molecular structures of water soluble BTE. This figure has been reproduced from ref. 92 with permission from the American Chemical Society.

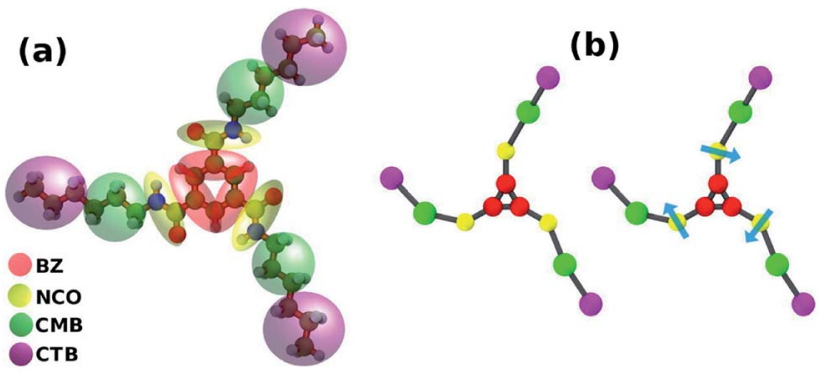

Fig. 18 (a) Coarse grain mapping of BTA and (b) the two CG models; the arrow represents a sticky dipole vector. This figure has been reproduced from ref. 56 with permission from the American Chemical Society

the hydrophobic part of the molecule. Upon shining light, the trans to cis isomerization of the azo group takes place, creating a strain in the tubule which causes its disassembly. Well tempered metadynamics based MD simulations have been able to realistically capture this phenomenon. ${ }^{95}$

\subsection{Coarse grain (CG) modelling}

An approach to perform MD simulations at equilibrium to study larger length and longer time scale phenomena than what is accessible via atomistic MD simulations are coarse graining (CG) methods. Herein, individual atoms are done away with and instead a collection of atoms (typically about three nonhydrogen atoms) are 'glued' to form a CG bead. Compared to atomistic models, CG models have fewer degrees of freedom, and have softer interactions which enable the use of larger MD timesteps of integration. In the domain of supramolecular polymer modelling, both these characteristics of CG approach enable the computational study of self-assembly at concentrations comparable to that in experiments, and the probing of defect mediated single molecule insertion into pre-organized stacks, without much loss of chemical specificity.

3.2.1 CG modelling in non-polar solvents. The cooperative nature of self-assembly of BTA in non-polar solvents was demonstrated using two CG models. ${ }^{56}$ In both the models Fig. 18, the phenyl ring was constructed out of three CG beads, to enable the representation of an aromatic plane. While three methylene groups on the self-assembling moiety constituted a CG bead, the linker amide was constructed either with one bead or with a bead plus a sticky point dipole in the other model. The sticky dipole on the amide bead contributes to the establishment of a macrodipole on the oligomer. The CG force field parameters were obtained by fitting the solvation and dimerization free energies obtained from atomistic MD simulations of BTA in a non-polar alkane solvent. The change in free energy of oligomers upon the addition of a monomer was calculated using the adaptive biasing force method for both the CG models. Both of them yielded a distinctive change in the slope of this quantity with oligomer size, a strong signature of the downhill cooperative nature of self-assembly of BTA and the size of the nucleus was found to be three. Furthermore, the 
model with the sticky dipole reproduced the formation of a macrodipole along the stack direction.

3.2.2 Polar solvents. In water soluble monomer units, at least the initial part of self-assembly is largely driven by hydrophobic interactions. ${ }^{\mathbf{9 1 , 9 2}}$ The hydrophobic effects are indicated by calculating the solvent accessible surface area (SASA), which will decrease from the value for dispersed monomers to those for stacks. SASA itself can be used as a reaction coordinate in free energy calculations in a pioneering work, Bochicchio and Pavan ${ }^{96}$ calculated the free energy surface of a water soluble BTA-CG model with respect to SASA and the number of hydrogen bonds per BTA which is shown in Fig. 19. The case study of dispered BTA in water reveals the fact that the amplification of the stacking order into long oligomers proceeds at a much lower rate following the fast initial aggregation process. The process of assembly and disassembly was studied from simulations at temperatures $27^{\circ} \mathrm{C}$ and $97^{\circ} \mathrm{C}$ respectively..$^{96}$

CG models have also been used to identify the increase in disorder of the supramolecular polymer formed by a fluorine substituted compound in comparison to its hydrogenated counterpart. ${ }^{98}$ While the latter is shown experimentally to self-assemble in a cooperative fashion, the former does so in an isodesmic manner.

The monomer exchange of molecule in a BTA stack solvated in linear alkane has been studied using a CG model. The free energy required to remove a molecule from the stack is independent of its position. ${ }^{56}$ The exact mechanism of monomer insertion into a stack in aqueous solution was demonstrated convincingly by Pavan and coworkers to be via defect locations (hot spots) on the stack. BTA exchange is a stepwise process as shown in Fig. 20(a). While the motion of a monomer from a hot spot on the stack to other regions on the stack itself occurs in the $\mu$ s timescale, its diffusion into bulk water happens in ms timescale, as seen from well tempered metadynamics based coarse grained MD simulations. ${ }^{97}$ The free energy profile for this process shown in Fig. 20(b) too exemplifies this step-wise behaviour.

As mentioned earlier, chiral molecules form more stable stacks than achiral ones. ${ }^{91}$ So, it is very important to understand monomer exchange phenomena on chiral molecules. The exchange rates of chiral monomers in corresponding fibers are

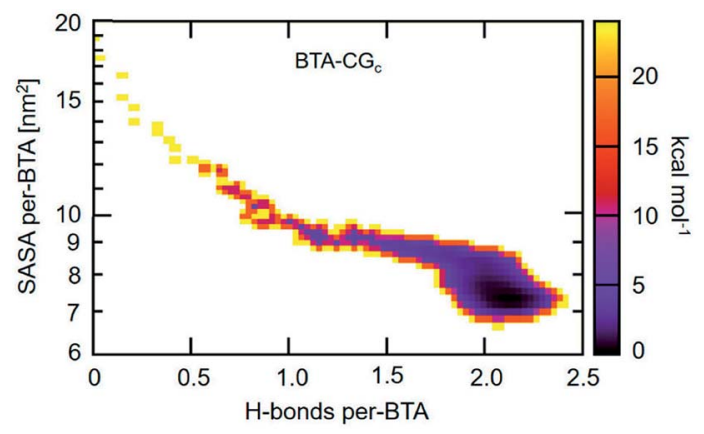

Fig. $192 \mathrm{D}$ self-assembly free energy landscape of BTA-CG model as a function of average number of $\mathrm{H}$-bonds per BTA and average SASA per BTA. This figure has been reproduced from ref. 96 with permission from the American Chemical Society.
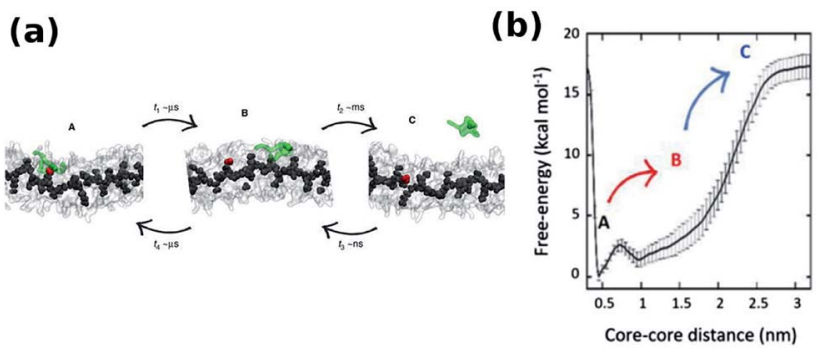

Fig. 20 (a) Snapshots of CG-BTA in water from well tempered MD representing state $A, B$ and $C$, (b) free-energy profile of monomer exchange with water as a solvent. ${ }^{97}$

slower than achiral ones by 1-2 orders of magnitude. The largest monomer movements occur in proximity of the fiber surface, while the internal core of the fibers appears more static. The rate of dynamic monomer exchange can be influenced by changing the monomer-monomer interaction or the H-bonding between the molecules. The change in interaction energy is proportional to the rate of monomer movements, and the lack of hydrogen bond increases monomer movements. ${ }^{97}$ These monomer exchange can be used for defect control in materials.

\section{Conclusions}

While supramolecular polymers have been experimentally studied for nearly two decades or more, their atomistic modelling have been attempted only over the past six to seven years or so. Yet, modelling has contributed several significant original results. Some of these include the identification of the $2: 1$ dipole configuration in the classical molecule, BTA, as the ground state, the reversal of helical handedness concomitant with polarization switching in BTA ferroelectrics, and the fine role of defects (hot spots) for monomer insertion into a supramolecular polymer. A variety of molecular modelling techniques, including gas phase quantum chemical methods, atomistic and coarse grained MD simulations have been employed. In view of the long timescales involved in the process of self-assembly, enhanced sampling methods such as adaptive biasing force, well tempered metadynamics, replica exchange solute tempering etc., have been of effectively employed. Yet, mesoscale ordering in terms of fiber formation and their properties have not been modelled. These might require solvent-free models ${ }^{99}$ and mesoscale methods such as dissipative particle dynamics. ${ }^{\mathbf{1 0 0 , 1 0 1}}$

It is also vital to derive transferable CG force fields for various moieties and functional groups used in supramolecular polymers, so that the structure and phenomena exhibited by such assemblies can be computed in near real time to interpret new experiments. This will form one of the immediate tasks in our research group.

\section{Conflicts of interest}

There are no conflicts to declare. 


\section{Acknowledgements}

D. B. K. thanks CSIR, India for a Senior Research Fellowship. We thank DST for support.

\section{Notes and references}

1 K. Wolf, H. Frahm and H. Harms, Z. Phys. Chem. (B), 1937, 36, 237-287.

2 F. Huang and E. V. Anslyn, Chem. Rev., 2015, 115, 69997000.

3 J.-M. Lehn, Angew. Chem., Int. Ed., 1988, 27, 89-112.

4 L. Brunsveld, B. Folmer, E. W. Meijer and R. Sijbesma, Chem. Rev., 2001, 101, 4071-4098.

5 O. J. Goor, J. E. Brouns and P. Y. Dankers, Polym. Chem., 2017, 8, 5228-5238.

6 F. Deflorian, S. Rossi and E. Scrinzi, Corros. Eng., Sci. Technol., 2013, 48, 147-154.

7 Q. Wei, C. Schlaich, S. Prévost, A. Schulz, C. Böttcher, M. Gradzielski, Z. Qi, R. Haag and C. A. Schalley, Adv. Mater., 2014, 26, 7358-7364.

8 S. P. Wijnands, W. Engelen, R. P. Lafleur, E. W. Meijer and M. Merkx, Nat. Commun., 2018, 9, 65.

9 R. Haag, Angew. Chem., Int. Ed., 2004, 43, 278-282.

10 Y. Cheng, A. C. Samia, J. D. Meyers, I. Panagopoulos, B. Fei and C. Burda, J. Am. Chem. Soc., 2008, 130, 10643-10647.

11 H. Gheybi and M. Adeli, Polym. Chem., 2015, 6, 2580-2615. 12 R. Timpl and J. C. Brown, BioEssays, 1996, 18, 123-132.

13 R. A. MacDonald, B. F. Laurenzi, G. Viswanathan, P. M. Ajayan and J. P. Stegemann, J. Biomed. Mater. Res., Part A, 2005, 74, 489-496.

14 P. Carol, S. Sreejith and A. Ajayaghosh, Chem.-Asian J., 2007, 2, 338-348.

15 C.-Z. Wang, J.-L. Chen, Y. Tang, Y. Zang, G.-R. Chen, T. D. James, J. Li, C. Wu and X.-P. He, ACS Appl. Mater. Interfaces, 2017, 9, 3272-3276.

16 R. Thirumalai, R. D. Mukhopadhyay, V. K. Praveen and A. Ajayaghosh, Sci. Rep., 2015, 5, 9842.

17 S. Dong, B. Zheng, F. Wang and F. Huang, Acc. Chem. Res., 2014, 47, 1982-1994.

18 X. Ji, Y. Yao, J. Li, X. Yan and F. Huang, J. Am. Chem. Soc., 2012, 135, 74-77.

19 S. Dong, B. Zheng, M. Zhang, X. Yan, X. Ding, Y. Yu and F. Huang, Macromolecules, 2012, 45, 9070-9075.

20 F. Wang, C. Han, C. He, Q. Zhou, J. Zhang, C. Wang, N. Li and F. Huang, J. Am. Chem. Soc., 2008, 130, 11254-11255.

21 M. M. Smulders, M. M. Nieuwenhuizen, T. F. de Greef, P. van der Schoot, A. P. Schenning and E. W. Meijer, Chem.-Eur. J., 2010, 16, 362-367.

22 X. Yan, T. R. Cook, J. B. Pollock, P. Wei, Y. Zhang, Y. Yu, F. Huang and P. J. Stang, J. Am. Chem. Soc., 2014, 136, 4460-4463.

23 Z. Zhang, Y. Luo, J. Chen, S. Dong, Y. Yu, Z. Ma and F. Huang, Angew. Chem., 2011, 123, 1433-1437.

24 R. B. Martin, Chem. Rev., 1996, 96, 3043-3064.
25 P. Besenius, G. Portale, P. H. Bomans, H. M. Janssen, A. R. Palmans and E. W. Meijer, Proc. Natl. Acad. Sci. U. S. A., 2010, 107, 17888-17893.

26 M. M. Smulders, P. J. Stals, T. Mes, T. F. Paffen, A. P. Schenning, A. R. Palmans and E. W. Meijer, J. Am. Chem. Soc., 2009, 132, 620-626.

27 K. V. Rao, D. Miyajima, A. Nihonyanagi and T. Aida, Nat. Chem., 2017, 9, 1133-1139.

28 S. Chen, S. M. Polen, L. Wang, M. Yamasaki, C. M. Hadad and J. D. Badjić, J. Am. Chem. Soc., 2016, 138, 11312-11317.

29 T.-Y. Zhou, Q.-Y. Qi, Q.-L. Zhao, J. Fu, Y. Liu, Z. Ma and X. Zhao, Polym. Chem., 2015, 6, 3018-3023.

30 W. Seo, K. L. Carpenter, J. A. Gaugler, W. Shao, K. A. Werling, P. M. Fournier, D. S. Lambrecht and A. Star, J. Polym. Sci., Part A: Polym. Chem., 2017, 55, 1095-1101.

31 J. Adisoejoso, Y. Li, J. Liu, P. N. Liu and N. Lin, J. Am. Chem. Soc., 2012, 134, 18526-18529.

32 H. Kar, D. W. Gehrig, N. K. Allampally, G. Fernández, F. Laquai and S. Ghosh, Chem. Sci., 2016, 7, 1115-1120.

33 C. Zhou, J. Tian, J.-L. Wang, D.-W. Zhang, X. Zhao, Y. Liu and Z.-T. Li, Polym. Chem., 2014, 5, 341-345.

34 L. Chen, S.-C. Zhang, H. Wang, Y.-M. Zhou, Z.-T. Li and D.-W. Zhang, Tetrahedron, 2014, 70, 4778-4783.

35 C.-W. Hu, T. Sato, J. Zhang, S. Moriyama and M. Higuchi, ACS Appl. Mater. Interfaces, 2014, 6, 9118-9125.

36 J. Tian, T.-Y. Zhou, S.-C. Zhang, S. Aloni, M. V. Altoe, S.-H. Xie, H. Wang, D.-W. Zhang, X. Zhao, Y. Liu, et al., Nat. Commun., 2014, 5, 5574.

37 K. Jayaramulu, P. Kanoo, S. J. George and T. K. Maji, Chem. Commun., 2010, 46, 7906-7908.

38 S. Cherumukkil, B. Vedhanarayanan, G. Das, V. K. Praveen and A. Ajayaghosh, Bull. Chem. Soc. Jpn., 2017, 91, 100-120.

39 M. Kumar and S. J. George, Nanoscale, 2011, 3, 2130-2133.

40 M. Kumar and S. J. George, Chem.-Eur. J., 2011, 17, 1110211106.

41 J. Guilleme, M. J. Mayoral, J. Calbo, J. Aragó, P. M. Viruela, E. Ortí, T. Torres and D. González-Rodríguez, Angew. Chem., Int. Ed., 2015, 54, 2543-2547.

42 P. P. Neelakandan, P. C. Nandajan, B. Subymol and D. Ramaiah, Org. Biomol. Chem., 2011, 9, 1021-1029.

43 J. Leira-Iglesias, A. Sorrenti, A. Sato, P. A. Dunne and T. M. Hermans, Chem. Commun., 2016, 52, 9009-9012.

44 P. J. Stals, M. M. Smulders, R. Martín-Rapún, A. R. Palmans and E. W. Meijer, Chem.-Eur. J., 2009, 15, 2071-2080.

45 R. Marty, R. Nigon, D. Leite and H. Frauenrath, J. Am. Chem. Soc., 2014, 136, 3919-3927.

46 Y. Nakano, T. Hirose, P. J. Stals, E. W. Meijer and A. R. Palmans, Chem. Sci., 2012, 3, 148-155.

47 C. Kulkarni, P. A. Korevaar, K. K. Bejagam, A. R. Palmans, E. W. Meijer and S. J. George, J. Am. Chem. Soc., 2017, 139, 13867-13875.

48 D. E. Fagnani, M. J. Meese, K. A. Abboud and R. K. Castellano, Angew. Chem., Int. Ed., 2016, 55, 1072610731.

49 K. K. Bejagam, G. Fiorin, M. L. Klein and S. Balasubramanian, J. Phys. Chem. B, 2014, 118, 5218-5228. 
50 D. B. Korlepara, K. K. Bejagam and S. Balasubramanian, J. Phys. Chem. B, 2017, 121, 11492-11503.

51 T. Metzroth, A. Hoffmann, R. Martín-Rapún, M. M. Smulders, K. Pieterse, A. R. Palmans, J. A. Vekemans, E. W. Meijer, H. W. Spiess and J. Gauss, Chem. Sci., 2011, 2, 69-76.

52 H. Anetai, Y. Wada, T. Takeda, N. Hoshino, S. Yamamoto, M. Mitsuishi, T. Takenobu and T. Akutagawa, J. Phys. Chem. Lett., 2015, 6, 1813-1818.

53 M. M. Smulders, A. P. Schenning and E. W. Meijer, J. Am. Chem. Soc., 2008, 130, 606-611.

54 A. Ajayaghosh, C. Vijayakumar, R. Varghese and S. J. George, Angew. Chem., 2006, 118, 470-474.

55 D. Bochicchio and G. M. Pavan, Adv. Phys.: X, 2018, 3, 1436408.

56 K. K. Bejagam and S. Balasubramanian, J. Phys. Chem. B, 2015, 119, 5738-5746.

57 J. Roosma, T. Mes, P. Leclère, A. R. Palmans and E. Meijer, J. Am. Chem. Soc., 2008, 130, 1120-1121.

58 C. Kulkarni, S. K. Reddy, S. J. George and S. Balasubramanian, Chem. Phys. Lett., 2011, 515, 226-230.

59 T. F. De Greef, M. M. Smulders, M. Wolffs, A. P. Schenning, R. P. Sijbesma and E. W. Meijer, Chem. Rev., 2009, 109, 5687-5754.

60 A. R. Palmans and E. W. Meijer, Angew. Chem., Int. Ed., 2007, 46, 8948-8968.

61 I. Urbanaviciute, X. Meng, T. D. Cornelissen, A. V. Gorbunov, S. Bhattacharjee, R. P. Sijbesma and M. Kemerink, Adv. Electron. Mater., 2017, 3, 1600530.

62 M. A. Veld, D. Haveman, A. R. Palmans and E. W. Meijer, Soft Matter, 2011, 7, 524-531.

63 X. Caumes, A. Baldi, G. Gontard, P. Brocorens, R. Lazzaroni, N. Vanthuyne, C. Troufflard, M. Raynal and L. Bouteiller, Chem. Commun., 2016, 52, 13369-13372.

64 A. Gorbunov, T. Putzeys, I. Urbanavičiūtè, R. Janssen, M. Wübbenhorst, R. Sijbesma and M. Kemerink, Phys. Chem. Chem. Phys., 2016, 18, 23663-23672.

65 A. Gorbunov, X. Meng, I. Urbanaviciute, T. Putzeys, M. Wübbenhorst, R. Sijbesma and M. Kemerink, Phys. Chem. Chem. Phys., 2017, 19, 3192-3200.

$66 \mathrm{~J}$. van Gestel, P. van der Schoot and M. Michels, Macromolecules, 2003, 36, 6668-6673.

67 A. Šarić, A. K. Buell, G. Meisl, T. C. Michaels, C. M. Dobson, S. Linse, T. P. Knowles and D. Frenkel, Nat. Phys., 2016, 12, 874.

68 T. M. Hermans, Nat. Nanotechnol., 2016, 11, 920.

69 C. Kulkarni, G. Periyasamy, S. Balasubramanian and S. J. George, Phys. Chem. Chem. Phys., 2014, 16, 1466114664.

70 M. J. Mayoral, C. Rest, V. Stepanenko, J. Schellheimer, R. Q. Albuquerque and G. Fernández, J. Am. Chem. Soc., 2013, 135, 2148-2151.

71 A. Desmarchelier, M. Raynal, P. Brocorens, N. Vanthuyne and L. Bouteiller, Chem. Commun., 2015, 51, 7397-7400.

72 K. K. Bejagam, R. C. Remsing, M. L. Klein and S. Balasubramanian, Phys. Chem. Chem. Phys., 2017, 19, 258-266.
73 Y. Liu, Y. Jia, E. Zhu, L. Liu, Y. Qiao, G. Che and B. Yin, New J. Chem., 2017, 41, 11060-11068.

74 D. Weiss, D. Skrybeck, H. Misslitz, D. Nardini, A. Kern, K. Kreger and H.-W. Schmidt, ACS Appl. Mater. Interfaces, 2016, 8, 14885-14892.

75 Y. Sun and Z. Li, Polym. Chem., 2017, 8, 4422-4427.

76 C. F. Fitié, W. C. Roelofs, M. Kemerink and R. P. Sijbesma, J. Am. Chem. Soc., 2010, 132, 6892-6893.

77 C. F. Fitié, W. C. Roelofs, P. C. Magusin, M. Wübbenhorst, M. Kemerink and R. P. Sijbesma, J. Phys. Chem. B, 2012, 116, 3928-3937.

78 K. K. Bejagam, C. Kulkarni, S. J. George and S. Balasubramanian, Chem. Commun., 2015, 51, 1604916052.

79 C. Kulkarni, K. K. Bejagam, S. P. Senanayak, K. Narayan, S. Balasubramanian and S. J. George, J. Am. Chem. Soc., 2015, 137, 3924-3932.

80 U. H. Hansmann, Chem. Phys. Lett., 1997, 281, 140-150.

81 Y. Sugita and Y. Okamoto, Chem. Phys. Lett., 1999, 314, 141151.

82 R. C. Bernardi, M. C. Melo and K. Schulten, Biochim. Biophys. Acta, 2015, 1850, 872-877.

83 C. Abrams and G. Bussi, Entropy, 2013, 16, 163-199.

84 P. Liu, B. Kim, R. A. Friesner and B. Berne, Proc. Natl. Acad. Sci. U. S. A., 2005, 102, 13749-13754.

85 X. Huang, M. Hagen, B. Kim, R. A. Friesner, R. Zhou and B. J. Berne, J. Phys. Chem. B, 2007, 111, 5405-5410.

86 L. Wang, R. A. Friesner and B. Berne, J. Phys. Chem. B, 2011, 115, 9431-9438.

87 H. H. Arefi and T. Yamamoto, J. Chem. Phys., 2017, 147, 211102.

88 J. Boekhoven and S. I. Stupp, Adv. Mater., 2014, 26, 16421659.

89 L. Albertazzi, D. van der Zwaag, C. M. Leenders, R. Fitzner, R. W. van der Hofstad and E. W. Meijer, Science, 2014, 344, 491-495.

90 L. Albertazzi, F. J. Martinez-Veracoechea, C. M. Leenders, I. K. Voets, D. Frenkel and E. W. Meijer, Proc. Natl. Acad. Sci. U. S. A., 2013, 110, 12203-12208.

91 M. B. Baker, L. Albertazzi, I. K. Voets, C. M. Leenders, A. R. Palmans, G. M. Pavan and E. W. Meijer, Nat. Commun., 2015, 6, 6234.

92 M. Garzoni, M. B. Baker, C. M. Leenders, I. K. Voets, L. Albertazzi, A. R. Palmans, E. W. Meijer and G. M. Pavan, J. Am. Chem. Soc., 2016, 138, 13985-13995.

93 A. Mishra, D. B. Korlepara, M. Kumar, A. Jain, N. Jonnalagadda, K. K. Bejagam, S. Balasubramanian and S. J. George, Nat. Commun., 2018, 9, 1295.

94 C.-S. Chen, X.-D. Xu, S.-Y. Li, R.-X. Zhuo and X.-Z. Zhang, Nanoscale, 2013, 5, 6270-6274.

95 J. W. Fredy, A. Méndez-Ardoy, S. Kwangmettatam, D. Bochicchio, B. Matt, M. C. Stuart, J. Huskens, N. Katsonis, G. M. Pavan and T. Kudernac, Proc. Natl. Acad. Sci. U. S. A., 2017, 114, 11850-11855.

96 D. Bochicchio and G. M. Pavan, ACS Nano, 2017, 11, 10001011. 
97 D. Bochicchio, M. Salvalaglio and G. M. Pavan, Nat. Commun., 2017, 8, 147.

98 N. M. Casellas, S. Pujals, D. Bochicchio, G. M. Pavan, T. Torres, L. Albertazzi and M. García-Iglesias, Chem. Commun., 2018, 54, 4112-4115.
99 D. Bochicchio and G. M. Pavan, J. Phys. Chem. Lett., 2017, 8, 3813-3819.

100 Y. Zhou, M. Huang, T. Lu and H. Guo, Macromolecules, 2018, 51, 3135-3148.

101 G. Kacar, Phys. Chem. Chem. Phys., 2018, 20, 12303-12311. 